\title{
Decompression Surgery versus Interspinous Devices for Lumbar Spinal Stenosis: A Systematic Review of the Literature
}

\author{
Jennifer Tram, Shanmukha Srinivas, Arvin R. Wali, Courtney S. Lewis, Martin H. Pham \\ Department of Neurosurgery, University of California San Diego School of Medicine, San Diego, CA, USA
}

\begin{abstract}
In this retrospective review study, the authors systematically reviewed the literature to elucidate the efficacy and complications associated with decompression and interspinous devices (ISDs) used in surgeries for lumbar spinal stenosis (LSS). LSS is a debilitating condition that affects the lumbar spinal cord and spinal nerve roots. However, a comprehensive report on the relative efficacy and complication rate of ISDs as they compare to traditional decompression procedures is currently lacking. The PubMed database was queried to identify clinical studies that exclusively investigated decompression, those that exclusively investigated ISDs, and those that compared decompression with ISDs. Only prospective cohort studies, case series, and randomized controlled trials that evaluated outcomes using the Visual Analog Scale (VAS), Oswestry Disability Index, or Japanese Orthopedic Association scores were included. A random-effects model was established to assess the difference between preoperative and the 1-2-year postoperative VAS scores between ISD surgery and lumbar decompression. This study included 40 papers that matched our criteria. Twenty-five decompressionexclusive clinical trials with 3,386 patients and a mean age of 68.7 years (range, 31-88 years) reported a 2.2\% incidence rate of dural tears and a $2.6 \%$ incidence rate of postoperative infections. Eight ISD-exclusive clinical trials with 1,496 patients and a mean age of 65.1 (range, 19-89 years) reported a 5.3\% incidence rate of postoperative leg pain and a 3.7\% incidence rate of spinous process fractures. Seven studies that compared ISDs and decompression in 624 patients found a reoperation rate of $8.3 \%$ in ISD patients vs. $3.9 \%$ in decompression patients; they also reported dural tears in $0.32 \%$ of ISD patients vs. $5.2 \%$ in decompression patients. A metaanalysis of the randomized controlled trials found that the differences in preoperative and postoperative VAS scores between the two groups were not significant. Both decompression and ISD interventions are unique surgical interventions with different therapeutic efficacies and complications. The collected studies do not consistently demonstrate superiority of either procedure over the other but understanding the differences between the two techniques can help tailor treatment regimens for patients with LSS.
\end{abstract}

Keywords: Lumbar spinal stenosis; Decompression surgery; Interspinous device; Interspinous device surgery

\section{Introduction}

Lumbar spinal stenosis is a compression of the cauda equina nerve roots secondary to narrowing of the central canal. Nerve compression occurs either directly as a result of surrounding structures or because increased intrathecal pressure and ischemia impede the function of spinal nerve roots, which results in pain, especially during standing or

\footnotetext{
Received Apr 6, 2019; Revised May 27, 2019; Accepted Jun 20, 2019

Corresponding author: Jennifer Tram

University of California, San Diego School of Medicine, P.O. 80421, 9450 Gilman Drive, La Jolla, CA 92092, USA

Tel: +1-949-350-2633, Fax: +1-714-546-6235, E-mail: jtram@ucsd.edu
} 
walking [1].

While lumbar spinal stenosis can be congenital [2], the most common cause of lumbar spinal stenosis is degenerative spondylosis [3], which commonly affects the elderly population. In fact, lumbar spinal stenosis is a leading cause of disability in the elderly [4-12] with reported rates as high as $11.2 \%$ in the $70-80$-year-old population. Moreover, lumbar spinal stenosis poses a significant socioeconomic burden to patients and was associated with $\$ 1.65$ billion in Medicare costs in 2009 [13]. Thus, judicious treatment of lumbar spinal stenosis can substantially decrease healthcare costs.

Laminectomy is the most commonly performed decompressive procedure in patients with lumbar spinal stenosis [14]. For patients without spondylolisthesis, interspinous devices (ISDs) offer a minimally invasive alternative treatment option to decompress the site of stenosis. These devices provide indirect decompression through posterior distraction of the spinous processes. To date, a comprehensive report on the relative efficacy and complication rate of ISDs as they compare with traditional decompression procedures has not been published. In this article, the authors systematically review the functional outcomes and complications from the use of decompression surgery and ISDs for lumbar spinal stenosis.

\section{Methods}

A systematic review was performed to analyze the outcomes from decompression surgery and ISDs using PubMed and included articles dating from December 1989 to August 2018. The search term used for the first part of this review was "Decompression surgery for lumbar spinal stenosis." The search term used for the second part of this review was "Interspinous devices for lumbar spinal stenosis." The search term used for the third part of this review was "Interspinous devices versus decompression for lumbar spinal stenosis." These searches yielded a total of 208 results. Of these, 20 articles were excluded due to insufficient data or inability to access full-text articles in English. Articles were included within this review if they presented primary human data on decompression surgery or ISDs for lumbar spinal stenosis. Only prospective cohort studies, case series, and randomized controlled trials that quantitatively evaluated outcomes using the Visual Analog Scale (VAS), Oswestry Disability Index (ODI), or Japanese Orthopedic Association (JOA) scores were included. Clinical studies that involved other surgical procedures were excluded. According to these criteria, 40 experimental studies met the inclusion criteria.

The extracted clinical articles were then divided into three categories, as follows: (1) studies that described only decompression (Fig. 1), (2) studies that described only ISDs (Fig. 2), and (3) studies that described both ISDs and decompression (Fig. 3). All studies were evaluated for sample size, average age, type of decompression surgery or ISD, functional recovery via VAS, ODI, or JOA score, complications, mortality, and average follow-up or followup range.

ProMeta 3 was used to generate a random-effects model to analyze the pooled VAS score outcomes obtained by randomized controlled trials that examined differences in preoperative and 1-2-year postoperative VAS pain scores. A positive effect size indicated that ISD surgery was associated with a greater reduction in VAS pain scores compared with lumbar decompression, whereas a negative effect size indicated that lumbar decompression was associated with a greater reduction in pain scores compared

Clinical trial manuscripts identified from December 1989 to September 2018 querying PubMed database for "Decompression surgery for lumbar spinal stenosis" ( $n=165)$

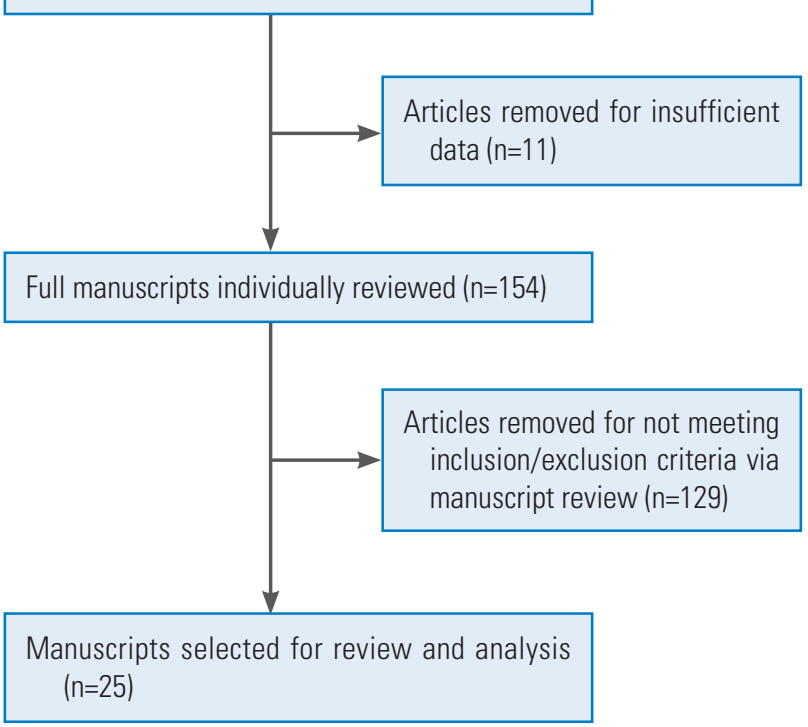

Fig. 1. Flowchart for screening manuscripts that reported "Decompression surgery for lumbar spinal stenosis." In all, 165 clinical studies were initially identified from the PubMed database. After the inclusion and exclusion criteria were applied, 25 manuscripts (11 randomized controlled trials, 11 prospective cohort studies, and three case series) were selected for review and analysis. 
31 Clinical trial manuscripts identified from December 1989 to September 2018 querying PubMed database for "Interspinous devices for lumbar spinal stenosis."

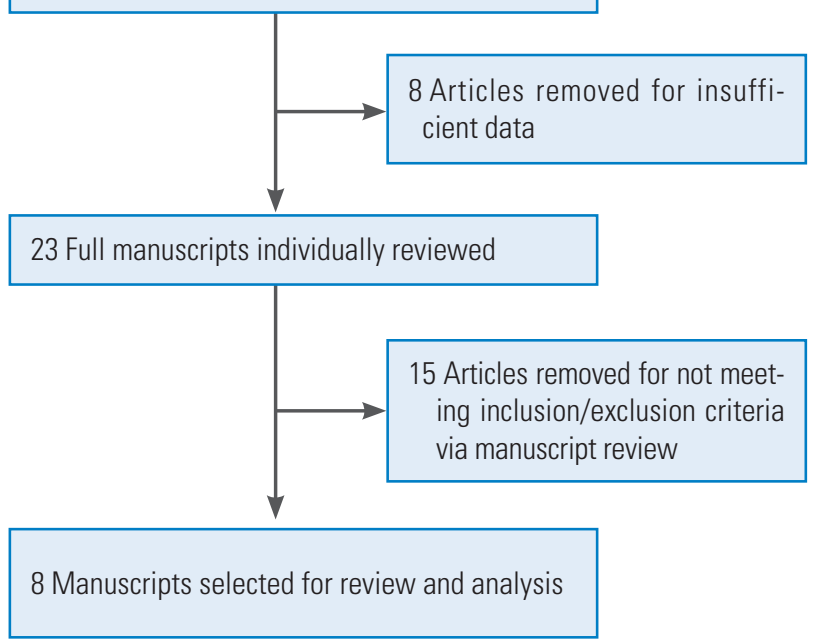

Fig. 2. Flowchart for screening manuscripts that reported "Interspinous devices for lumbar spinal stenosis." Thirty-one clinical studies that investigated the role of interspinous device for lumbar spinal stenosis were initially identified in the PubMed database. After the inclusion and exclusion criteria were applied, eight manuscripts (four randomized controlled trials, three prospective cohort studies, and one case series) were selected for review and analysis.

13 Clinical trial manuscripts identified from December 1989 to September 2018 querying PubMed database for "Interspinous devices versus decompression for lumbar spinal stenosis" ( $n=5)$. Relevant manuscripts from search 1 were added $(n=7)$. Relevant manuscripts from search 1 were added $(n=1)$.

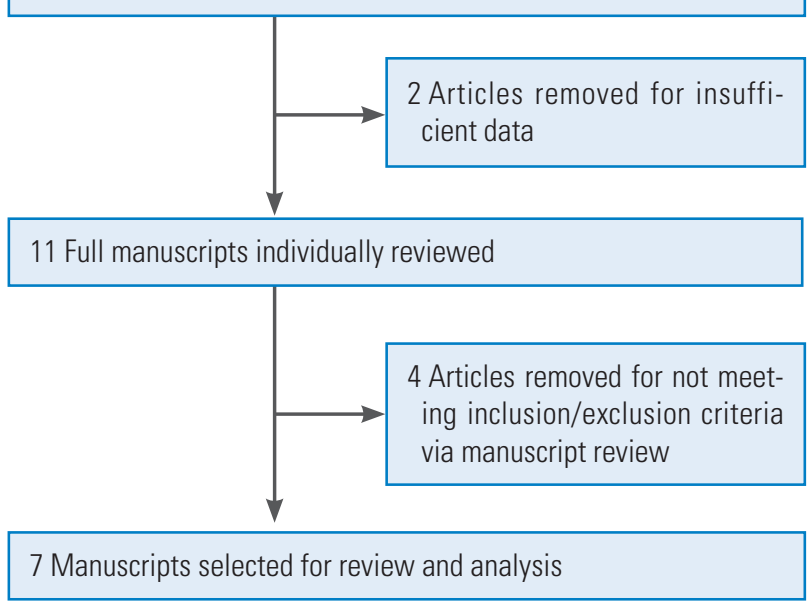

Fig. 3. Flowchart for screening manuscripts that reported "Interspinous devices versus decompression for lumbar spinal stenosis." Twelve clinical studies that compared interspinous device with decompression for lumbar spinal stenosis were initially identified in the PubMed database, and eight relevant studies from previous queries were added. After the inclusion and exclusion criteria were applied, seven manuscripts (one prospective cohort study and six randomized controlled clinical trials) were selected for review and analysis. with ISD surgery. The longest available postoperative data presented in a given study were used for this analysis. If articles only presented differences between preoperative and postoperative VAS pain scores rather than raw data for postoperative pain scores, the standard deviation for those differences in pain scores was used to model the standard deviation of the postoperative pain scores. A $p$ value less than 0.05 was considered statistically significant.

\section{Results}

\section{Studies exclusive to decompression}

Twenty-five studies that singularly described the use of decompression surgery for lumbar spinal stenosis were included. In all, these studies involved 3,386 patients, including 1,395 males and 1,895 females, whereas two studies did not report the gender $[15,16]$. The average participant age was 68.7 years (range, $31-88$ years). Types of decompression surgery included Marmot operation [17], minimally invasive [4-12], traditional laminectomy [15-25], unilateral laminectomy [16], open laminectomy [8], endoscopic interlaminar approach [9], endoscopic unilateral decompression [9], lumbar spinous process splitting, midline decompression, mild percutaneous decompression [26], mild interlaminar decompression, windows technique laminoforaminotomy [22], bilateral laminotomy [25], and unilateral laminotomy [16,19,25,27]. The average follow-up period for these studies was 17.6 months (range, 0-90 months). The major findings included decreased neuropathic pain as measured by VAS [4,9,10,15,19-24,26-29], decreased functional impairment secondary to lower back pain as measured by ODI [6$11,16,18,21-23,26,27,29]$, and reduction in severity of lower back pain as measured by JOA score $[4,5,11,19,28]$ (Table 1). Studies also reported complications associated with decompression such as dural tear $[5,8,9,11,12,16,19,21$ 24] $(\mathrm{n}=65 / 3,386,2.2 \%)$, transient neuralgia $[5,7,9,11,12]$ $(n=26 / 3,386,0.77 \%)$, hematoma at the epidural or peridural levels $[5,8,9,16,23,25](\mathrm{n}=16 / 3,386,0.47 \%)$, infection (respiratory, wound, urinary tract) $[5,8,11,18,21,22,25]$ $(\mathrm{n}=87 / 3,386,2.6 \%)$, procedural hemorrhage $[6,7,18]$ $(\mathrm{n}=3 / 3,386,0.089 \%)$, allergy [18] $(\mathrm{n}=2 / 3,386,0.059 \%)$, micturition problems $[8,9,20](\mathrm{n}=27 / 3,386,0.80 \%)$, cardiovascular complications including myocardial infarction, pulmonary emboli, and deep vein thromboses $[8,21](n=5 / 3,386,0.15 \%)$, foot drop [9] $(n=3 / 3,386$, 


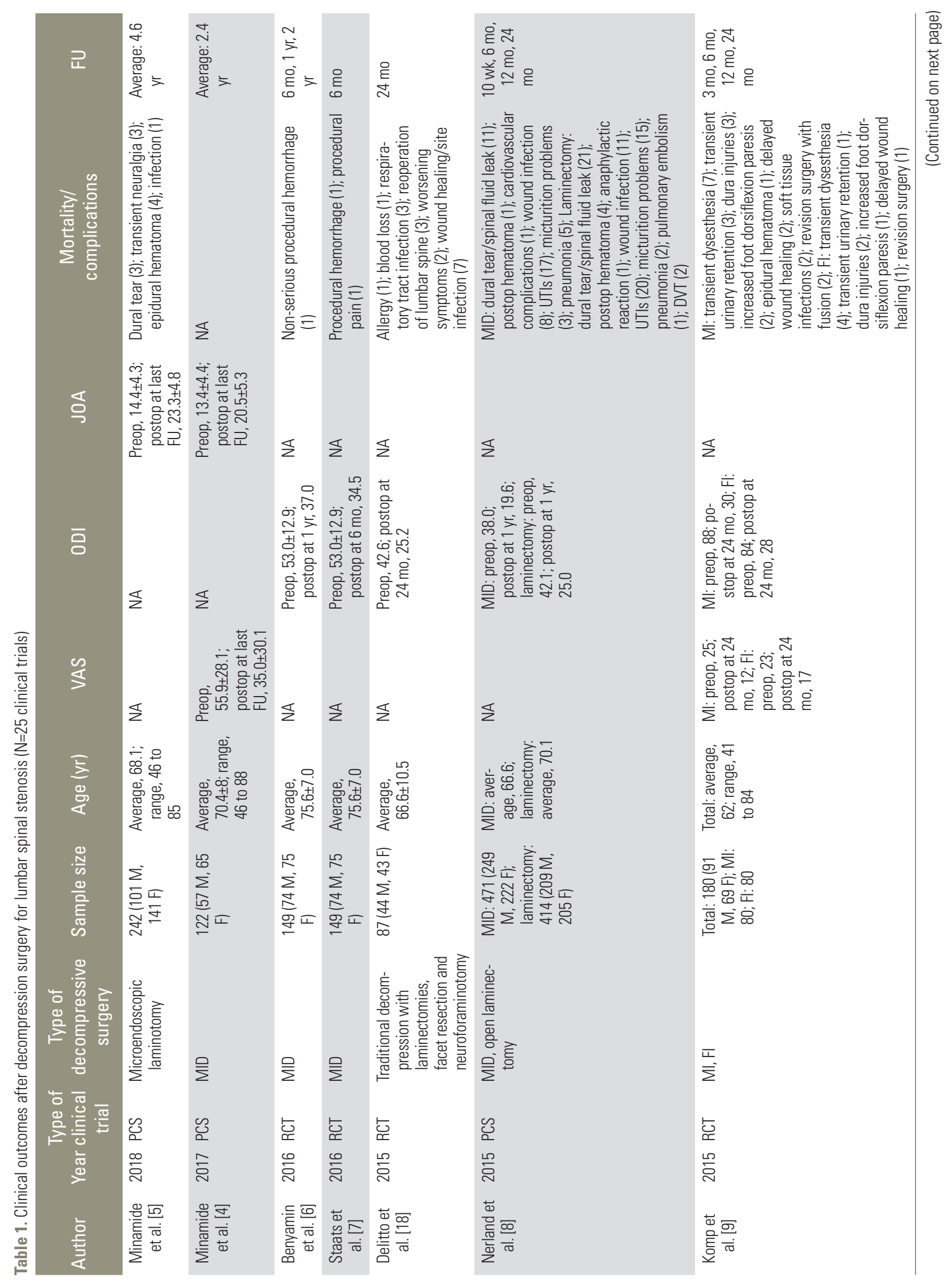




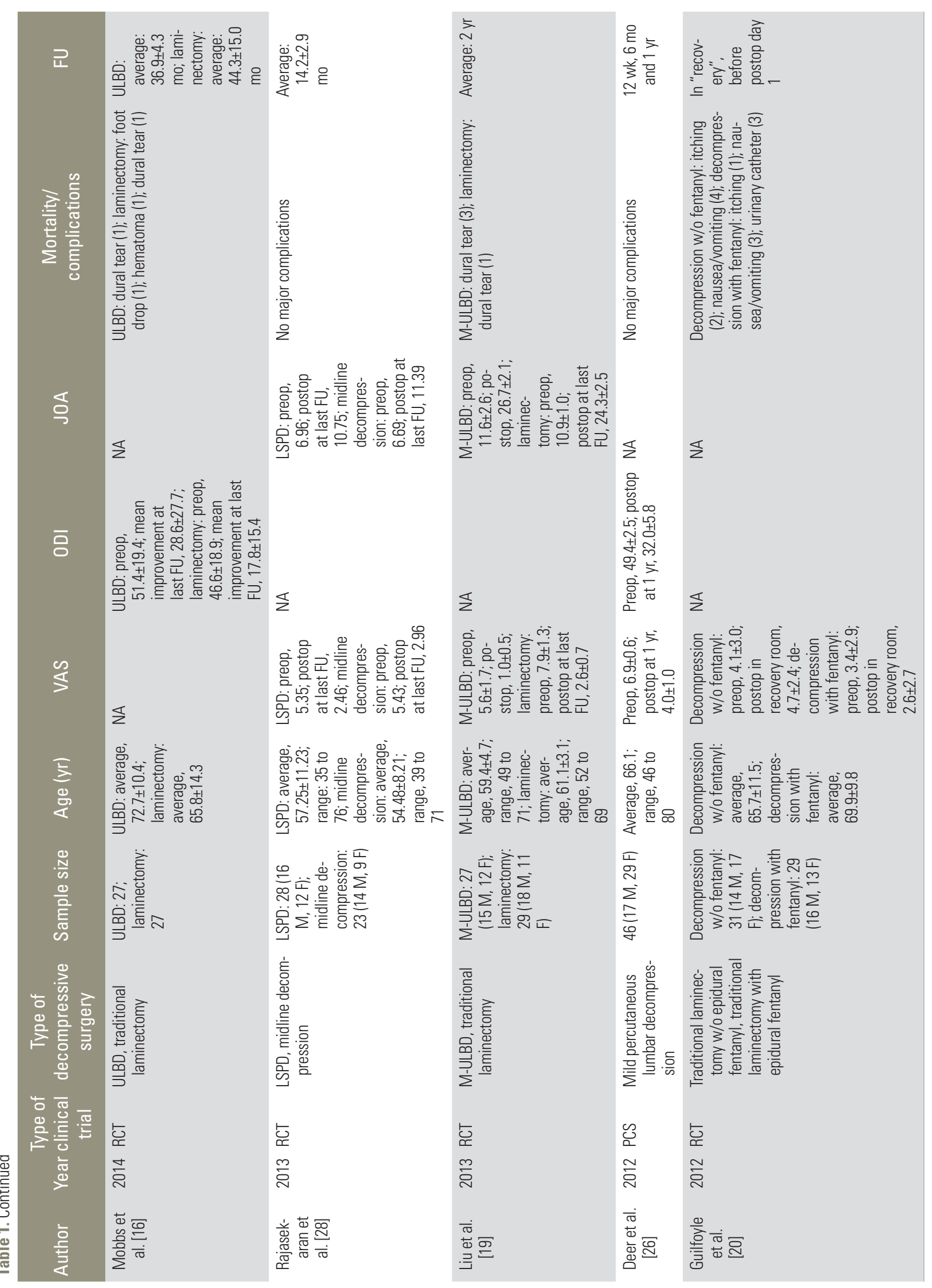



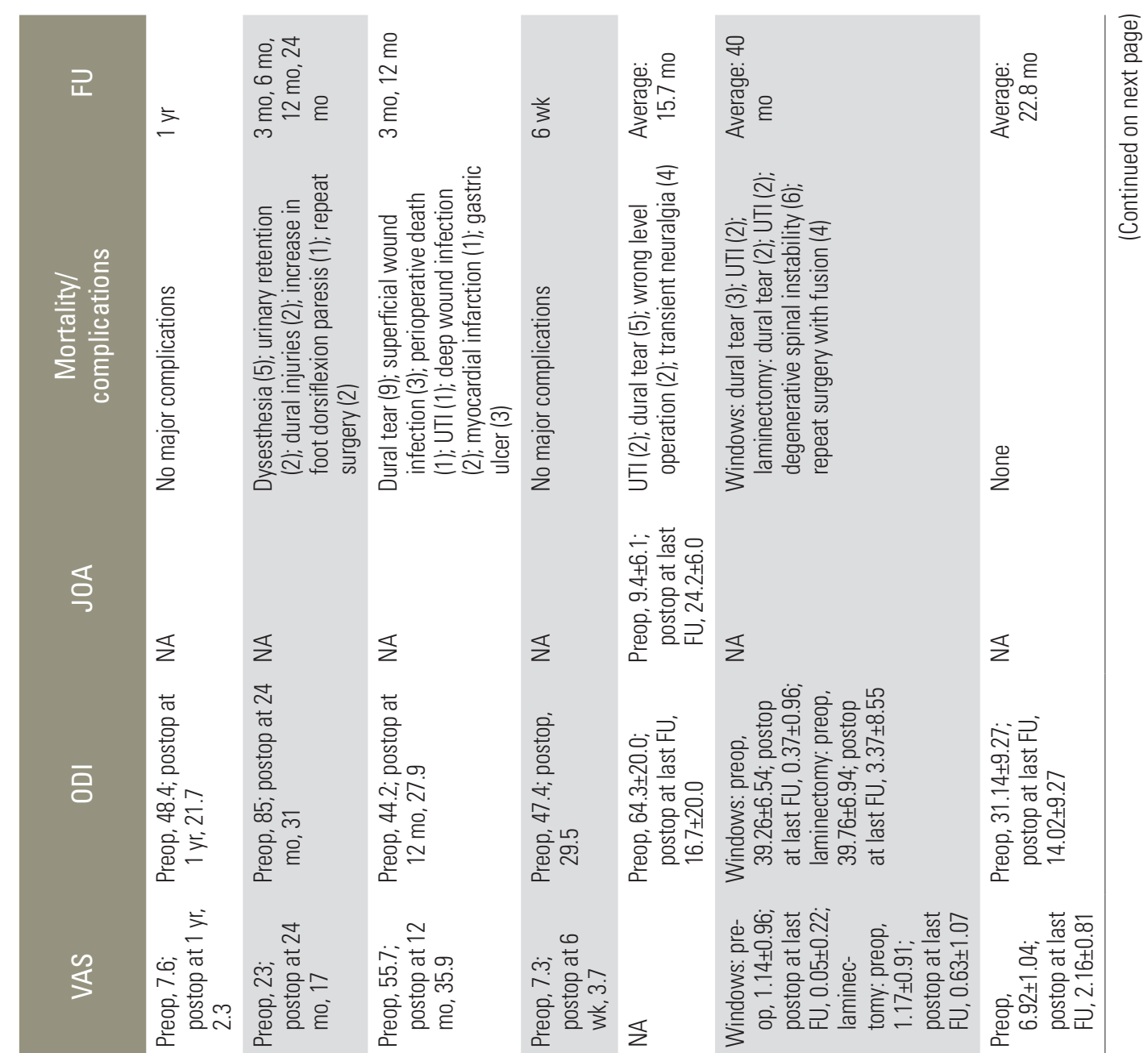

ఏ

$\overline{0}$

$\overline{0}$

$\sum$

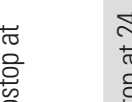

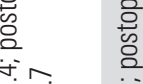

ờ

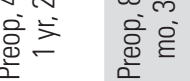

这是

$\stackrel{4}{>}$

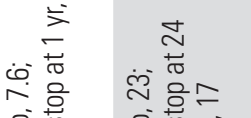

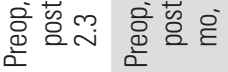

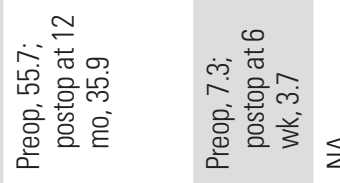

$\stackrel{0}{\overline{5}}$

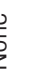

$\Sigma$

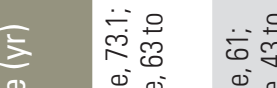

인

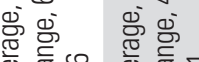

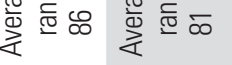

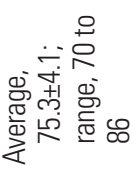

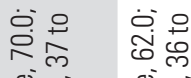

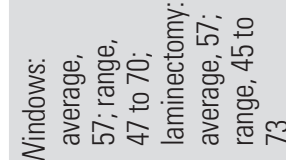

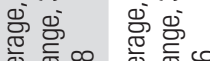

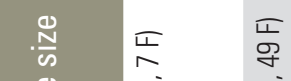

in

인

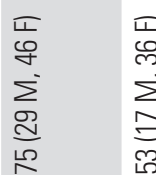

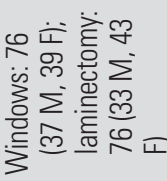

군

㚙要

ले 을 市

을

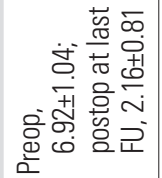

낟요 웅

ब.

实

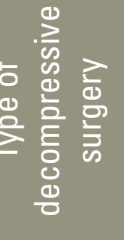
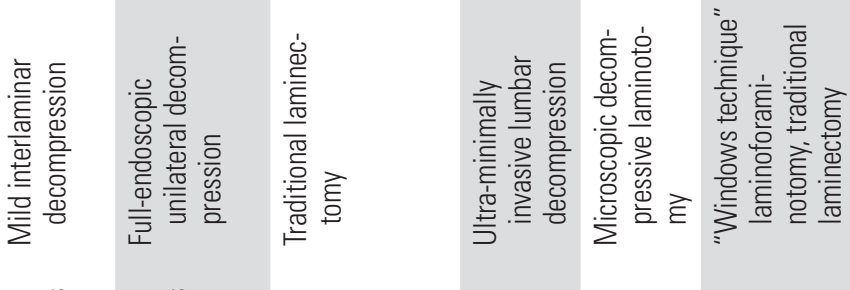

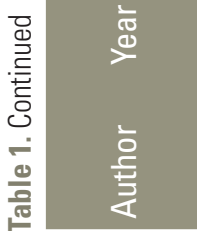

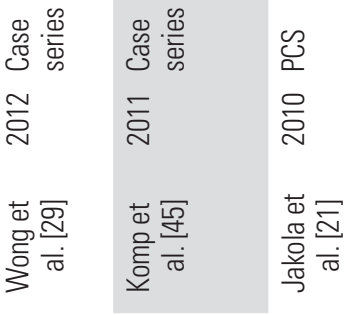

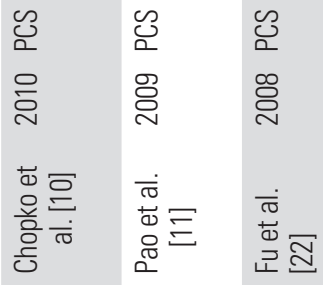

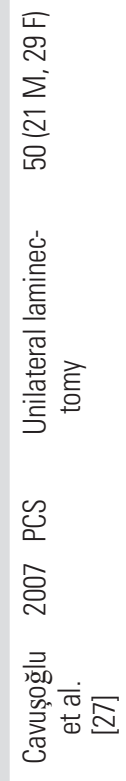




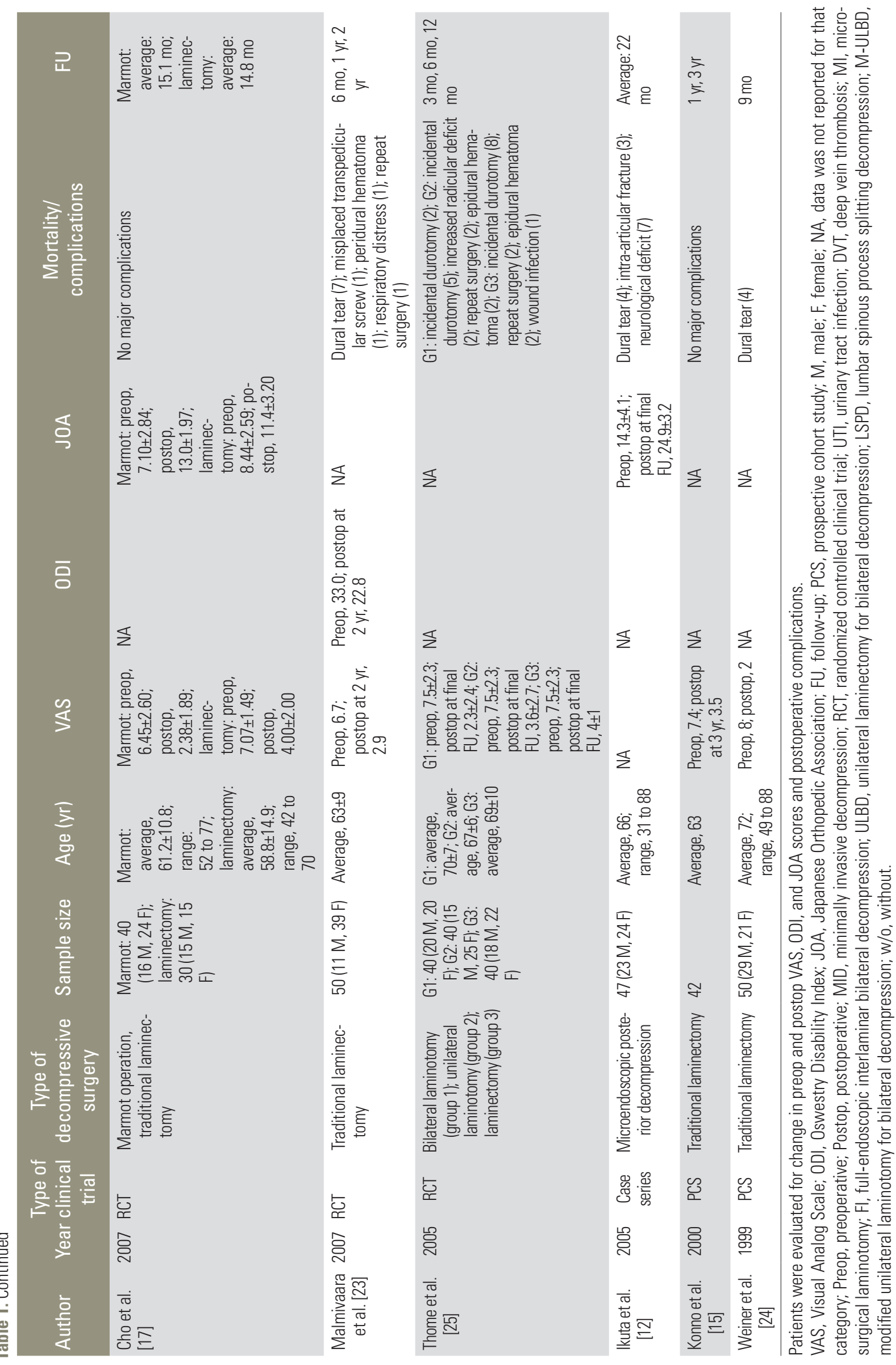


$0.089 \%)$, pruritus [20] $(\mathrm{n}=3 / 3,386,0.089 \%)$, nausea and vomiting [20] $(n=7 / 3,386,0.21 \%)$, gastric ulcers [21] $(\mathrm{n}=3 / 3,386,0.089 \%)$, and degenerative spinal instability [22] $(n=6 / 3,386,0.18 \%)$. Reoperation was required in a few cases $[9,18,22,23,25](n=16 / 3,386,0.47 \%)$. Only one study reported a perioperative fatality caused by myocardial infarction 1 month after surgery [21].

\section{Studies exclusive to interspinous devices}

Eight studies that singularly described the use of ISDs for lumbar spinal stenosis were included. In all, these studies involved 1,496 patients, including 807 males and 578 females, whereas two studies did not report the gender [30,31]. The average participant age was 65.1 years (range, 19-89 years). The types of ISDs included percutaneous interspinous process spacer [32] $(n=80 / 1,496,5.3 \%)$, Superion $[31,33,34](n=404 / 1,496,27.0 \%), X$-stop [30,3336] $(n=911 / 1,496,60.9 \%)$, and Aperius $(n=156 / 1,496$, $10.4 \%)$. The average follow-up time was 23.1 months (range, 1-84 months). The major quantitative findings of ISD treatment for lumbar stenosis included reduction of the VAS score $[30,31,33,35,37]$ and a decrease in the ODI score $[30,31,33,34,36]$ (Table 2). Unlike in the decompression studies, the JOA score was not used to evaluate changes after ISD use. Complications resulting from ISDs were postoperative back pain [34,37] $(n=101 / 1,496,6.8 \%)$, leg pain including sciatica [34,37] $(n=80 / 1,496,5.3 \%)$, spinous process fracture $[32,34,35,37]$ $(n=56 / 1,496,3.7 \%)$, buttock/groin pain [34] $(n=22 / 1,496$, $1.5 \%)$, cerebrospinal fluid leakage [35] ( $n=9 / 1,496,0.60 \%)$, infection including in both superficial skin and deep wounds $[33,35](n=15 / 1,496,1.0 \%)$, device dislocation $[32,35](n=19 / 1,496,1.3 \%)$, reoperation [33] $(n=18 / 1,496$, $1.2 \%)$, removal of the ISD $(n=14 / 1,496,0.94 \%)$, hematoma $(\mathrm{n}=1 / 1,496,0.07 \%)$, and spinal claudication [37] $(n=3 / 1,496,0.20 \%)$.

\section{Studies that compared interspinous device and de- compression}

Seven studies compared the efficacy of decompression versus that of ISD for lumbar spinal stenosis through a randomized controlled trial design or a prospective clinical study. In all, 624 patients were included in these studies: 310 underwent decompression and 314 received an ISD. The average age could not be calculated because some studies included a median age instead of an average age $[38,39]$. Approaches for decompression included minimally invasive [40], primary lumbosacral [41] spinal bony decompression [38], posterior midline approach [42], and traditional $[39,43,44]$. The types of ISDs used included Coflex [43], X-stop [40,43,44], Wallis implant [41], interspinous dynamic stabilization devices [42], and a foraminal enlargement lumbar interspinous distraction device [39]. In one study, the type of ISD used was not specified [38]. The average follow-up time in these studies was 18.9 months (range, 0-40 months).

All seven clinical trials demonstrated a statistically significant decrease from the preoperative VAS or ODI scores to the postoperative VAS or ODI scores within each treatment group [38-44]. With regards to improvement in neuropathic pain as measured by the VAS, some studies showed greater improvements between preoperative and the last follow-up after decompression compared with ISD surgery $[38,43,44]$. With regards to back pain-induced functional abnormalities, one study showed greater improvement between preoperative and the last follow-up after decompression compared with ISD [1]. However, one study showed the opposite effect [40,43] (Table 3). Differences in complication rates between decompression and ISDs were variable in each study. While some studies showed a higher rate of reoperation with ISDs $[38,43]$, others showed the opposite [44] or similar reoperation rates [40]. Dural tears were almost exclusively seen in the decompression group [38-41,43,44], with only a single study reporting one incident of a dural tear in the ISD group [1]. Spinous process fractures were also exclusively seen in the ISD group, with no reported fractures in any of the decompression groups [38-41,43,44] (Table 4).

Several other important factors in the selection of the surgical procedure are the reoperation rates, estimated blood loss, and operative times. Not every clinical trial reported reoperation rates, and for the data that were collected, the results were mixed in terms of the differences in the reoperation rates between decompression surgery and ISD insertion surgery. A $0.47 \%$ reoperation rate was found in the studies exclusive to decompression surgery $[9,18,22,23,25]$ and a $1.2 \%$ reoperation rate was found in the studies exclusive to ISD surgery [33]. However, studies directly comparing the two treatments reported a $3.9 \%$ reoperation rate with decompression surgery and a $8.3 \%$ reoperation rate with ISDs $[38,40,43,44]$. Similarly, estimated blood loss and operation times were inconsis- 


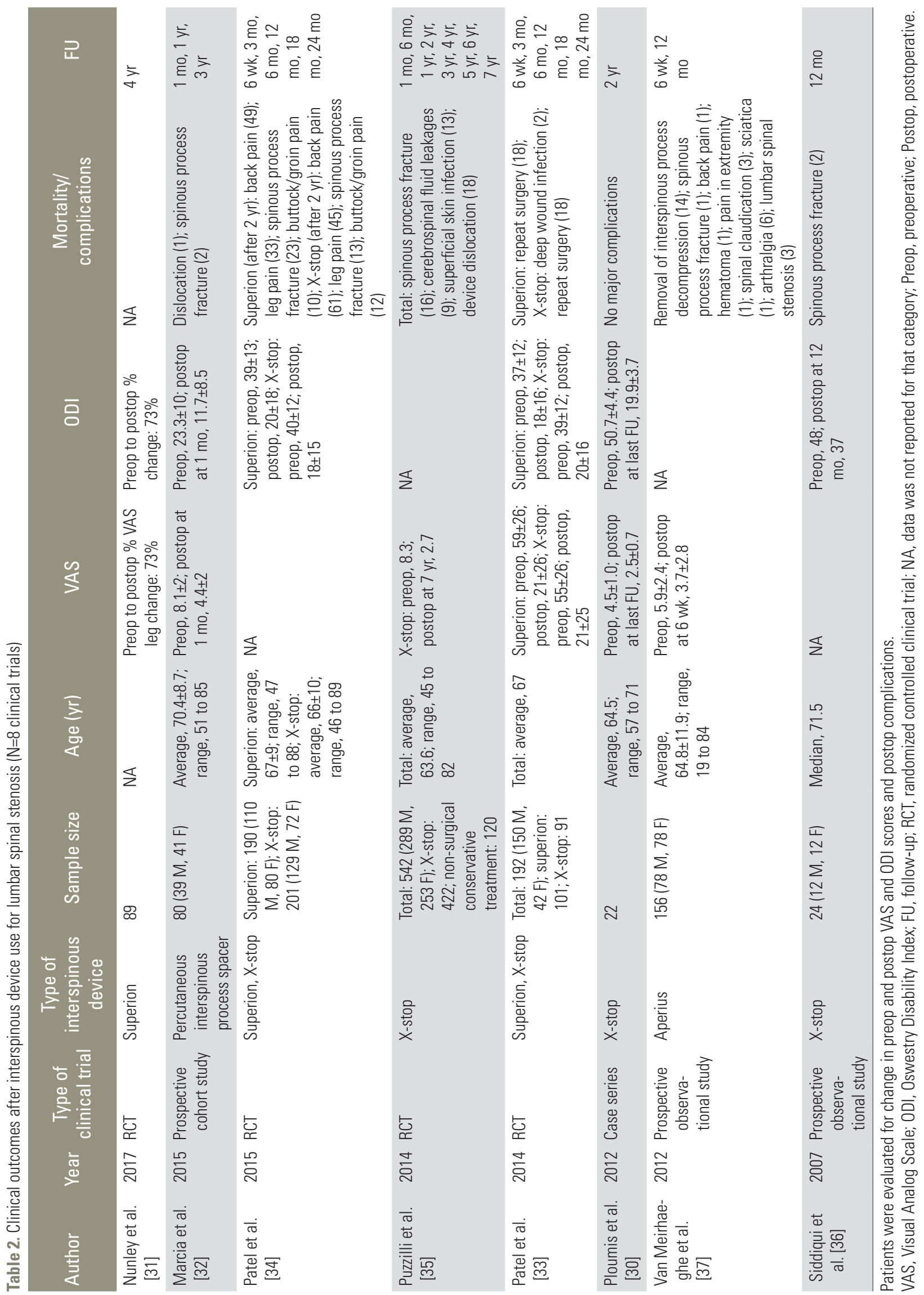




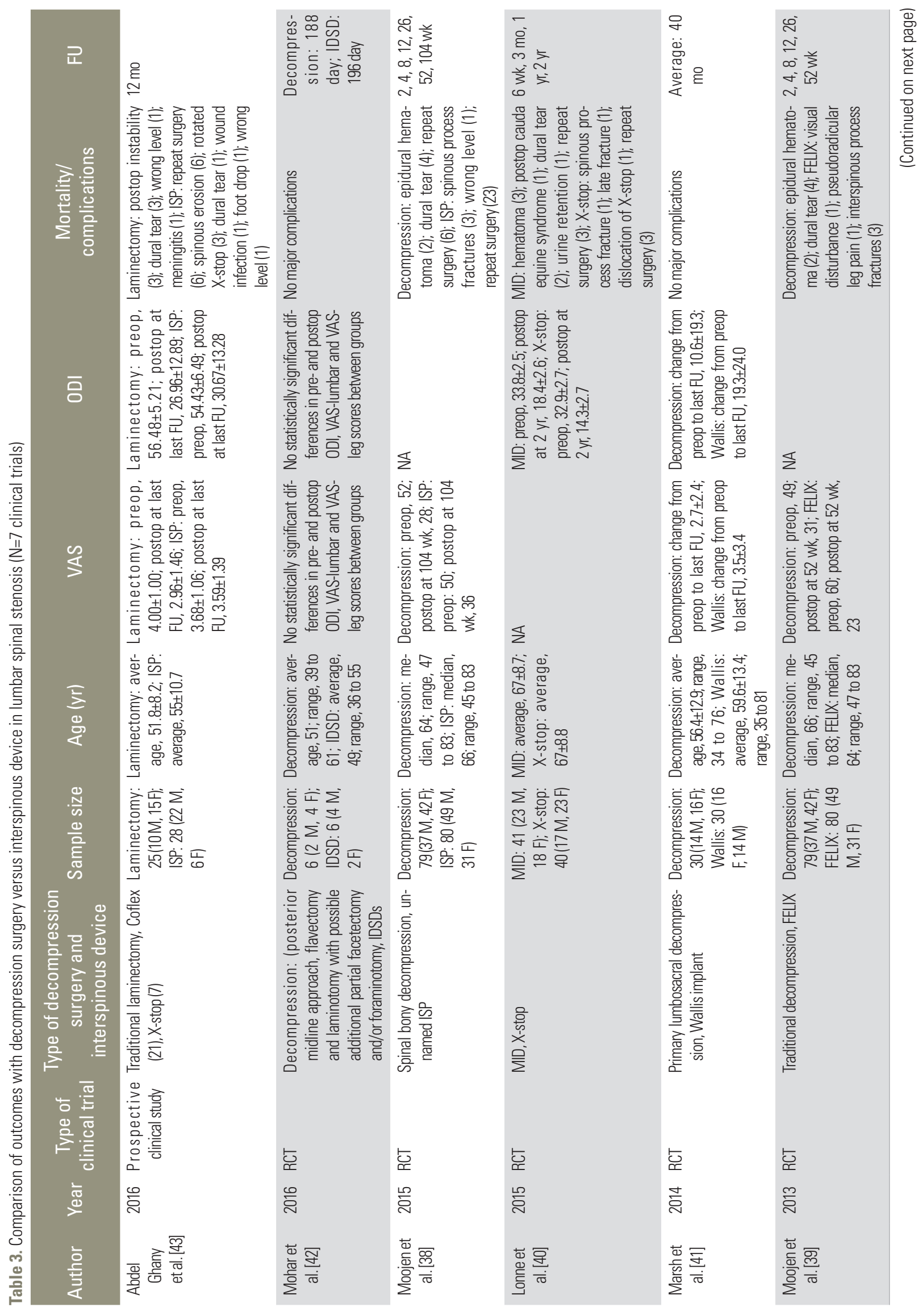




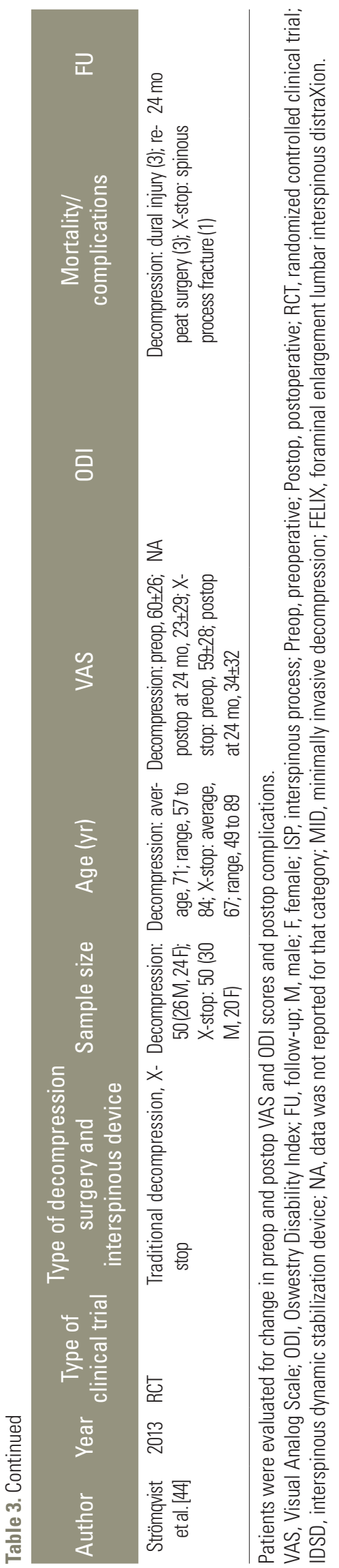

tently reported in the clinical trials that were reviewed. The average operation time in decompression-only trials was $92.30 \pm 54.78$ minutes [8]. The average operation time in ISD-only trials was $43.33 \pm 8.50$ minutes [37]. In trials that directly compared decompression surgery and ISD surgery, the average operation time was $77.50 \pm 41.44$ minutes for the former procedure [37] and 57.55 \pm 45.44 minutes for the latter procedure [37]. The average intraoperative blood loss in decompression-only studies was $110.18 \pm 88.74 \mathrm{~mL}$ [38]. The average intraoperative blood loss in ISD-only studies was $23.33 \pm 17.56 \mathrm{~mL}$ [37]. In trials that directly compared decompression surgery and ISD surgery, the average intraoperative blood loss for the former procedure was $262 \pm 0 \mathrm{~mL}$ [37] and $143.07 \pm 125.96$ $\mathrm{mL}$ [37] for the latter procedure. Two studies provided categorized data rather than numerical data for blood loss, but in both studies, a characteristic of decompression trials was greater blood loss compared with ISD surgery [37].

\section{Meta-analysis}

Four randomized controlled trials assessed the differences in the preoperative and 1-2-year postoperative VAS scores between ISD surgery and lumbar decompression [38-41,43,44]. According to a random-effects model, the difference between the preoperative and pooled 1-2year postoperative VAS scores between ISD surgery and lumbar decompression was not statistically significant ( $p=0.166)$. The results from the random-effects model are graphically displayed as a forest plot in Fig. 4.

\section{Discussion}

This comprehensive systematic review investigated three groups of studies that examined the clinical and functional impact of decompression surgery or placement of ISDs for lumbar spinal stenosis. Of the literature that explored the role of decompression exclusively, most studies compiled within this review involved conventional decompression surgery via open laminectomy $[8,15,16,18-24,27]$ or laminotomy $[5,9,11,25]$, but other types of decompression surgery were explored, including minimally invasive decompression [4,6-8], full-endoscopic interlaminar bilateral decompression [9], the Marmot operation [17], lumbar spinous process splitting decompression, midline decompression, modified unilateral laminotomy for bi- 
Table 4. Complications associated with decompression-exclusive surgery, ISP-exclusive surgery, and ISP versus decompression surgeries

\begin{tabular}{|c|c|c|c|c|}
\hline \multirow{2}{*}{ Complication } & \multirow{2}{*}{$\begin{array}{l}\text { Decompression } \\
\text { surgery exclusive } \\
\text { studies }\end{array}$} & \multirow{2}{*}{$\begin{array}{c}\text { ISP device } \\
\text { exclusive studies }\end{array}$} & \multicolumn{2}{|c|}{ ISP vs. decompression surgeries } \\
\hline & & & Decompression & ISP device \\
\hline Dural tear & $65 / 3,386$ (2.2) & & $16 / 310(5.2)$ & 1/314 (0.32) \\
\hline Transient neuralgia & $26 / 3,386(0.77)$ & & & \\
\hline Hematoma at epidural or peridural level & $16 / 3,386(0.47)$ & $1 / 1,496(0.07)$ & $7 / 310(2.3)$ & \\
\hline Infection (respiratory, wound, or urinary tract infection) & $87 / 3,386(2.6)$ & $15 / 1,496(1.0)$ & & $1 / 314(0.32)$ \\
\hline Procedural hemorrhage & $3 / 3,386(0.089)$ & & & \\
\hline Allergy & $2 / 3,386(0.059)$ & & & \\
\hline Micturition problem & $27 / 3,386(0.80)$ & & & \\
\hline $\begin{array}{l}\text { Cardiovascular complication (myocardial infarction, } \\
\text { pulmonary emboli, deep vein thrombosis) }\end{array}$ & $5 / 3,386(0.15)$ & & & \\
\hline Increased foot dorsiflexion & 3/3,386 (0.089) & & & \\
\hline Pruritus & $3 / 3,386(0.089)$ & & & \\
\hline Nausea and vomiting & $7 / 3,386(0.21)$ & & & \\
\hline Gastric ulcers & $3 / 3,386(0.089)$ & & & \\
\hline Degenerative spinal instability & $6 / 3,386(0.18)$ & & & \\
\hline Wrong level of operation or misplaced screw & $3 / 3,386(0.089)$ & & $1 / 310(0.32)$ & $3 / 314(0.10)$ \\
\hline Reoperation & $16 / 3,386(0.47)$ & $18 / 1,496(1.2)$ & $12 / 310(3.9)$ & $26 / 314(8.3)$ \\
\hline Back pain & & $101 / 1,496(6.8)$ & & \\
\hline Buttock/groin pain & & $22 / 1,496(1.5)$ & & \\
\hline Spinal claudication & & $3 / 1,496(0.20)$ & & \\
\hline Leg pain & & $80 / 1,496(5.3)$ & & $1 / 314(0.32)$ \\
\hline Spinous process fracture & & $56 / 1,496(3.7)$ & & $9 / 314(2.9)$ \\
\hline Cerebrospinal fluid leakage & & $9 / 1,496(0.60)$ & & \\
\hline Device dislocation & & $19 / 1,496(1.3)$ & & $1 / 314(0.32)$ \\
\hline
\end{tabular}

Values are presented as number/total number (\%).

ISP, interspinous process.

Abdel et al. [43] (2016)

Marsh et al. [41] (2014)

Moojen et al. [38] (2015)

Stromqvist et al. [44] (2013)

Overall (random-effects model)

$\begin{array}{rrr}\text { ES } & \text { Sig. } & N \\ -0.73 & 0.009 & 53 \\ 0.30 & 0.244 & 60 \\ -2.35 & 0.000 & 160 \\ -0.45 & 0.026 & 100 \\ -0.81 & 0.166 & 373\end{array}$

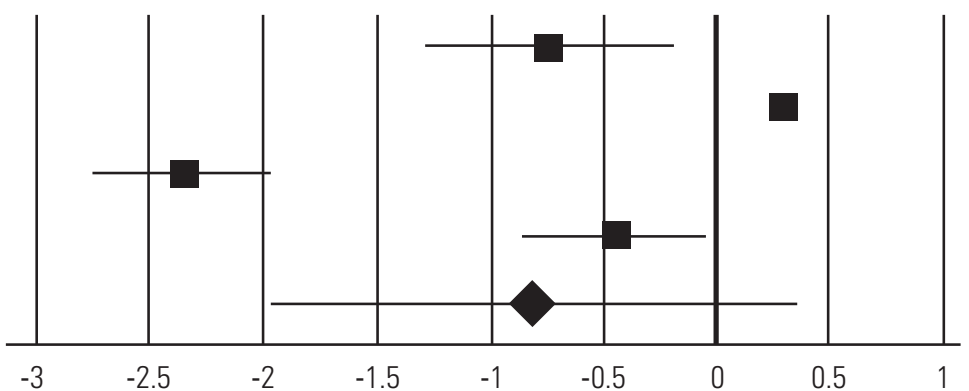

Fig. 4. Forest plot depicting the random-effects model of the differences in preoperative and postoperative VAS scores between ISD use and lumbar decompression. After pooling outcomes from the four randomized trials with VAS outcomes data, a statistically significant difference between the preoperative and 1-2-year postoperative VAS scores between ISD and lumbar decompression was not identified ( $p=0.166)$. A positive effect size indicates a greater reduction in VAS scores in the ISD group compared with the lumbar decompression group, while a negative effect size indicates a greater reduction in VAS scores in the lumbar decompression group compared with the ISD group. VAS, Visual Analog Scale; ISD, interspinous device; ES, effect size; Sig, statistical significance. 
lateral decompression, percutaneous minimally invasive lumbar decompression [26], "Windows" decompression [22], and ultra-minimally invasive lumbar decompression [10]. Minimally invasive decompression surgery is associated with comparable decreases in ODI scores as is open laminectomy, but this procedure caused fewer surgical complications including dural tears, postoperative hematomas, wound infections, and UTIs according to these studies [8]. Generally, regardless of the decompressive surgical approach, studies consistently demonstrated improvement in VAS, ODI, and JOA scores. Infection was the most common complication in clinical studies that exclusively examined decompression surgery $[5,8,9,18,21]$.

Papers that reviewed the use of ISDs for the surgical treatment of lumbar spinal stenosis mainly used the following types: the Superion, X-stop, percutaneous interspinous process spacers, and Aperius devices. Of these, Xstop was reviewed the most (five clinical studies) [30,3336], while Superion, ISPs, and Aperius were reviewed in three [31,33,34], one [32], and one [37], respectively. Both the Superion and X-stop devices showed comparable decreases in ODI and VAS scores, and varying similarities and differences were observed in postoperative complications. In several studies, X-stop cases were associated with a higher percentage of back and leg pain and wound infections but fewer incidences of spinous process fractures [34]. Of the two, the Superion device allows for a more minimally invasive approach and thus reduced disruption of neighboring spinal structures, which may account for the reduced postoperative pain observed in these patients [34]. Additionally, the Superion device has thicker wings than the X-stop device, which may increase long-term stability and reduce incidences of repeat surgery [34]. Across all studies that directly compared the two ISD devices, the Superion and X-stop implants had similar clinical outcomes, which suggests that preference for one surgery over the other might be indicated by surgeon familiarity and preference $[33,34]$. Complications and patient complaints necessitated the removal of nearly $10 \%$ of all Aperius implants in one clinical study. The most common complication within ISD-exclusive surgeries was spinous process fracture, which may be improved with increased surgeon familiarity and experience with placing the ISD [32,34-37].

In the seven studies that directly compared decompression surgery with ISD insertion, no clear consensus as to the superiority of either surgical intervention was reported. All studies showed statistically significant improvement in postoperative VAS and ODI scores within both treatment arms [38-44]. Across all six studies, the VAS and ODI scores were also comparable between the two groups; neither decompression nor ISD surgery consistently demonstrated superiority in either VAS or ODI scores. Three clinical studies demonstrated a greater change in VAS or ODI scores for decompression patients $[38,43,44]$, two clinical trials demonstrated a greater change in VAS scores for ISD patients $[39,40]$, and two clinical trials demonstrated no significant difference in the changes in VAS or ODI scores for decompression versus ISD patients [41,42]. One study reported 17 more repeat surgeries in the group that received ISD device insertion surgery than in the group that received decompression surgery, even with nearly equal patient sizes [38]. However, another study reported fewer incidences of repeat surgery and postoperative complications in the $\mathrm{X}$-stop group than in the decompression group. Some discrepancies were reported in the complications among studies. One study with a patient size of 60 mentioned no major complications, while other studies mentioned unique complications such as postoperative cauda equina syndrome and foot drop, which did not occur as a result of any other similar surgeries $[40,41,43]$. Similarly, the most common complication within clinical trials that examined decompression and ISD device insertion was reoperation $[38,40,43,44]$. A higher reoperation rate was observed in the ISD group compared with the decompression group, which suggests that decompression surgery has more favorable long-term results, at least in terms of this metric. Similarly, the ISD-exclusive studies reported a higher reoperation rate than the decompression surgery-exclusive studies. The heterogeneity in outcomes data between ISDs and lumbar decompression was reflected within our randomeffects model. While overall postoperative reductions in VAS scores were identified between ISDs and lumbar decompression, our model did not find a statistical difference between ISD surgery and lumbar decompression to cause a greater reduction in VAS scores compared with the other intervention.

Operation times and intraoperative blood loss can also be compared. The average operation time was consistently lower for ISD surgery than for decompression surgery, which may be beneficial for patients who cannot tolerate 
long operation times [8]. This conclusion was confirmed by the clinical trials that directly compared decompression surgery and ISD surgery. In all cases, these clinical trials reported longer operation times for decompression surgery [37]. Similarly, every clinical trial that directly compared the two treatment mechanisms reported less intraoperative bleeding for ISDs than for decompression surgery [37]. Incidentally, one clinical trial reported no measurable blood loss for decompression surgery primarily because there was continuous irrigation during surgery and thus no drainage was required $[8,45]$. Therefore, ISD surgery may be recommended for patients who benefit from shorter operation times and/or who are at greater risk for blood loss complications.

A concern with the use of ISDs is their potential to introduce segmental kyphosis through an increase in interspinous flexion and distraction as the mechanism of indirect decompression. This has led to significant concerns on how placement of ISDs can potentially negatively affect overall sagittal balance. Several biomechanical studies have been performed to investigate segmental tilt after implantation of an ISD [46-48]. Wilke et al. [47] found that while the DIAM device introduced kyphosis into the operated segment, the Coflex, Wallis, and X-stop devices had no effect on the $0.5^{\circ}-0.7^{\circ}$ of kyphosis. Another biomechanical study by Anasetti et al. [48] reported similar findings, as the DIAM device induced a shift toward kyphosis at the implanted level. Alfieri et al. [49] performed a systematic review of clinical studies that involved ISD and described the risk of introducing kyphosis at the operated lumbar segment. They identified 11 studies where kyphosis was measured preoperatively and postoperatively and reported no differences in the segmental angle. They concluded that no clinical data suggested that ISD significantly introduces kyphosis. Schulte et al. [50] has performed the only prospective study that evaluated sagittal balance in 20 patients who received an X-stop device. Using postoperative $91.44-\mathrm{cm}$ films, they found an average improvement in the overall sagittal alignment of -2.0 $\mathrm{cm}$. Their hypothesis was that a postoperative relief of stenosis was achieved, which allowed the spine to assume the most efficient posture and sagittal balance through the entirety of the lumbar spine, with only a segmental reduction in extension at the operated level. Although ISDs have a potential locally segmental kyphotic effect, there appears to be the potential for improvement in neurogenic posture from relief of claudication symptoms that may maintain overall global sagittal alignment.

In this review, we attempt to distill studies on decompression surgery or placement of an ISD for lumbar spinal stenosis as they relate to postoperative outcome and complication rates. The theoretical benefit of an ISD over traditional decompression lies in the potential for a more minimally invasive approach with a motion-preserving implant to relieve the symptoms of neurogenic claudication. ISDs may also be helpful in patients who cannot tolerate lengthy procedures under general anesthesia due to medical comorbidities. ISD surgery was consistently associated with shorter operation times and less intraoperative blood loss compared with decompression surgery, both in decompression-only and interspinous-only papers and in papers that assessed both treatment types. For prospective, technique-exclusive, or comparison studies, we found that ISD surgery leads to favorable postoperative functional outcomes in terms of VAS and ODI scores but that it does not demonstrate superiority over decompression surgery. Incidences of $5.3 \%, 1.3 \%$, and $8.3 \%$ in postoperative leg pain, device dislocation, and reoperation rate, respectively, were observed. Overall, we recommend the use of decompression surgery based on the lower reoperation rates. Lower reoperation rates were consistently reported in the decompression surgery-exclusive papers and the papers that compared ISD versus decompression surgery.

Limitations of this study include inconsistent reporting of measurements among studies. Inconsistencies were also found in the extent of complications reported, with more exhaustive studies reporting unique complications, while some studies simply stated that no major complications were encountered [10,15,26,29,30,41,42]. Another limitation of this paper is the variation in postoperative care, which is important for long-term complications such as reoperation rates. Despite these limitations, we believe the information provided in this review puts into perspective the overall functional outcomes and reported complication rates in prospective studies of decompression versus ISD surgery for lumbar spinal stenosis. As with all clinical treatments, careful patient selection with an appropriately tailored surgical intervention remains crucial for successful patient outcomes. 


\section{Conclusions}

Treatment of lumbar spinal stenosis can be accomplished through decompression surgery or through the insertion of an ISD. Although studies reported higher rates of dural tears in decompression surgery and higher reoperation rates after ISD surgery, a meta-analysis revealed no difference in functional outcome, as improvements were seen in both groups. Careful patient selection remains crucial for either surgical procedure to ensure optimal surgical outcomes tailored to each patient. More diverse studies are needed to determine the superiority of one technique over the other for different patient populations.

\section{Conflict of Interest}

No potential conflict of interest relevant to this article was reported.

\section{References}

1. Hall S, Bartleson JD, Onofrio BM, Baker HL Jr, Okazaki H, O'Duffy JD. Lumbar spinal stenosis: clinical features, diagnostic procedures, and results of surgical treatment in 68 patients. Ann Intern Med 1985;103:271-5.

2. Binder DK, Schmidt MH, Weinstein PR. Lumbar spinal stenosis. Semin Neurol 2002;22:157-66.

3. Atlas SJ, Delitto A. Spinal stenosis: surgical versus nonsurgical treatment. Clin Orthop Relat Res 2006;443:198-207.

4. Minamide A, Yoshida M, Iwahashi H, et al. Minimally invasive decompression surgery for lumbar spinal stenosis with degenerative scoliosis: predictive factors of radiographic and clinical outcomes. J Orthop Sci 2017;22:377-83.

5. Minamide A, Yoshida M, Simpson AK, et al. Minimally invasive spinal decompression for degenerative lumbar spondylolisthesis and stenosis maintains stability and may avoid the need for fusion. Bone Joint J 2018;100-B:499-506.

6. Benyamin RM, Staats PS, MiDAS Encore I. MILD(R) is an effective treatment for lumbar spinal stenosis with neurogenic claudication: MiDAS ENCORE randomized controlled trial. Pain Physician 2016;19:22942.

7. Staats PS, Benyamin RM; MiDAS ENCORE Inves- tigators. MiDAS ENCORE: randomized controlled clinical trial report of 6-month results. Pain Physician 2016;19:25-38.

8. Nerland US, Jakola AS, Solheim O, et al. Minimally invasive decompression versus open laminectomy for central stenosis of the lumbar spine: pragmatic comparative effectiveness study. BMJ 2015;350:h1603.

9. Komp M, Hahn P, Oezdemir S, et al. Bilateral spinal decompression of lumbar central stenosis with the full-endoscopic interlaminar versus microsurgical laminotomy technique: a prospective, randomized, controlled study. Pain Physician 2015;18:61-70.

10. Chopko B, Caraway DL. MiDAS I (mild decompression alternative to open surgery): a preliminary report of a prospective, multi-center clinical study. Pain Physician 2010;13:369-78.

11. Pao JL, Chen WC, Chen PQ. Clinical outcomes of microendoscopic decompressive laminotomy for degenerative lumbar spinal stenosis. Eur Spine J 2009;18:672-8.

12. Ikuta K, Arima J, Tanaka T, et al. Short-term results of microendoscopic posterior decompression for lumbar spinal stenosis: technical note. J Neurosurg Spine 2005;2:624-33.

13. Deyo RA, Mirza SK, Martin BI, Kreuter W, Goodman DC, Jarvik JG. Trends, major medical complications, and charges associated with surgery for lumbar spinal stenosis in older adults. JAMA 2010;303:125965.

14. Weinstein JN, Tosteson TD, Lurie JD, et al. Surgical versus nonsurgical therapy for lumbar spinal stenosis. N Engl J Med 2008;358:794-810.

15. Konno S, Kikuchi S. Prospective study of surgical treatment of degenerative spondylolisthesis: comparison between decompression alone and decompression with Graf system stabilization. Spine (Phila Pa 1976) 2000;25:1533-7.

16. Mobbs RJ, Li J, Sivabalan P, Raley D, Rao PJ. Outcomes after decompressive laminectomy for lumbar spinal stenosis: comparison between minimally invasive unilateral laminectomy for bilateral decompression and open laminectomy: clinical article. J Neurosurg Spine 2014;21:179-86.

17. Cho DY, Lin HL, Lee WY, Lee HC. Split-spinous process laminotomy and discectomy for degenerative lumbar spinal stenosis: a preliminary report. J Neurosurg Spine 2007;6:229-39. 
18. Delitto A, Piva SR, Moore CG, Welch WC. Surgery versus nonsurgical treatment of lumbar spinal stenosis. Ann Intern Med 2015;163:397-8.

19. Liu X, Yuan S, Tian Y. Modified unilateral laminotomy for bilateral decompression for lumbar spinal stenosis: technical note. Spine (Phila Pa 1976) 2013;38:E732-7.

20. Guilfoyle MR, Mannion RJ, Mitchell P, Thomson S. Epidural fentanyl for postoperative analgesia after lumbar canal decompression: a randomized controlled trial. Spine J 2012;12:646-51.

21. Jakola AS, Sorlie A, Gulati S, Nygaard OP, Lydersen S, Solberg T. Clinical outcomes and safety assessment in elderly patients undergoing decompressive laminectomy for lumbar spinal stenosis: a prospective study. BMC Surg 2010;10:34.

22. Fu YS, Zeng BF, Xu JG. Long-term outcomes of two different decompressive techniques for lumbar spinal stenosis. Spine (Phila Pa 1976) 2008;33:514-8.

23. Malmivaara A, Slatis P, Heliovaara M, et al. Surgical or nonoperative treatment for lumbar spinal stenosis?: a randomized controlled trial. Spine (Phila $\mathrm{Pa}$ 1976) 2007;32:1-8.

24. Weiner BK, Fraser RD, Peterson M. Spinous process osteotomies to facilitate lumbar decompressive surgery. Spine (Phila Pa 1976) 1999;24:62-6.

25. Thome C, Zevgaridis D, Leheta O, et al. Outcome after less-invasive decompression of lumbar spinal stenosis: a randomized comparison of unilateral laminotomy, bilateral laminotomy, and laminectomy. J Neurosurg Spine 2005;3:129-41.

26. Deer TR, Kim CK, Bowman RG 2nd, Ranson MT, Yee BS. Study of percutaneous lumbar decompression and treatment algorithm for patients suffering from neurogenic claudication. Pain Physician 2012;15:45160.

27. Cavusoglu H, Turkmenoglu O, Kaya RA, et al. Efficacy of unilateral laminectomy for bilateral decompression in lumbar spinal stenosis. Turk Neurosurg 2007;17:100-8

28. Rajasekaran S, Thomas A, Kanna RM, Prasad Shetty A. Lumbar spinous process splitting decompression provides equivalent outcomes to conventional midline decompression in degenerative lumbar canal stenosis: a prospective, randomized controlled study of 51 patients. Spine (Phila Pa 1976) 2013;38:1737-43.

29. Wong WH. Mild interlaminar decompression for the treatment of lumbar spinal stenosis: procedure description and case series with 1-year follow-up. Clin J Pain 2012;28:534-8.

30. Ploumis A, Christodoulou P, Kapoutsis D, Gelalis I, Vraggalas V, Beris A. Surgical treatment of lumbar spinal stenosis with microdecompression and interspinous distraction device insertion: a case series. J Orthop Surg Res 2012;7:35.

31. Nunley PD, Patel VV, Orndorff DG, Lavelle WF, Block JE, Geisler FH. Superion interspinous spacer treatment of moderate spinal stenosis: 4-year results. World Neurosurg 2017;104:279-83.

32. Marcia S, Hirsch JA, Chandra RV, et al. Midterm clinical and radiologic outcomes after percutaneous interspinous spacer treatment for neurogenic intermittent claudication. J Vasc Interv Radiol 2015;26:1687-93.

33. Patel VV, Whang PG, Haley TR, et al. Two-year clinical outcomes of a multicenter randomized controlled trial comparing two interspinous spacers for treatment of moderate lumbar spinal stenosis. BMC Musculoskelet Disord 2014;15:221.

34. Patel VV, Whang PG, Haley TR, et al. Superion interspinous process spacer for intermittent neurogenic claudication secondary to moderate lumbar spinal stenosis: two-year results from a randomized controlled FDA-IDE pivotal trial. Spine (Phila Pa 1976) 2015;40:275-82.

35. Puzzilli F, Gazzeri R, Galarza M, et al. Interspinous spacer decompression (X-STOP) for lumbar spinal stenosis and degenerative disk disease: a multicenter study with a minimum 3-year follow-up. Clin Neurol Neurosurg 2014;124:166-74.

36. Siddiqui M, Smith FW, Wardlaw D. One-year results of X Stop interspinous implant for the treatment of lumbar spinal stenosis. Spine (Phila Pa 1976) 2007;32:1345-8.

37. Van Meirhaeghe J, Fransen P, Morelli D, et al. Clinical evaluation of the preliminary safety and effectiveness of a minimally invasive interspinous process device APERIUS(R) in degenerative lumbar spinal stenosis with symptomatic neurogenic intermittent claudication. Eur Spine J 2012;21:2565-72.

38. Moojen WA, Arts MP, Jacobs WC, et al. IPD without bony decompression versus conventional surgical decompression for lumbar spinal stenosis: 2-year results of a double-blind randomized controlled trial. 
Eur Spine J 2015;24:2295-305.

39. Moojen WA, Arts MP, Jacobs WC, et al. Interspinous process device versus standard conventional surgical decompression for lumbar spinal stenosis: randomized controlled trial. BMJ 2013;347:f6415.

40. Lonne G, Johnsen LG, Rossvoll I, et al. Minimally invasive decompression versus $\mathrm{x}$-stop in lumbar spinal stenosis: a randomized controlled multicenter study. Spine (Phila Pa 1976) 2015;40:77-85.

41. Marsh GD, Mahir S, Leyte A. A prospective randomised controlled trial to assess the efficacy of dynamic stabilisation of the lumbar spine with the Wallis ligament. Eur Spine J 2014;23:2156-60.

42. Mohar J, Cimerman M. Radiological changes after interspinous dynamic stabilisation for lateral stenosis of lumbar spinal canal: a parallel group randomised trial. Acta Chir Orthop Traumatol Cech 2016;83:2638.

43. Abdel Ghany W, Amer A, Saeed K, et al. Evaluation of interspinous spacer outcomes in degenerative lumbar canal stenosis: clinical study. World Neurosurg 2016;95:556-64.

44. Stromqvist BH, Berg S, Gerdhem P, et al. X-stop versus decompressive surgery for lumbar neurogenic intermittent claudication: randomized controlled trial with 2-year follow-up. Spine (Phila Pa 1976) 2013;38:1436-42.

45. Komp M, Hahn P, Merk H, Godolias G, Ruetten S. Bilateral operation of lumbar degenerative central spinal stenosis in full-endoscopic interlaminar technique with unilateral approach: prospective 2 -year results of 74 patients. J Spinal Disord Tech 2011;24:281-7.

46. Parchi PD, Evangelisti G, Vertuccio A, et al. Biomechanics of interspinous devices. Biomed Res Int 2014;2014:839325.

47. Wilke HJ, Drumm J, Haussler K, Mack C, Steudel WI, Kettler A. Biomechanical effect of different lumbar interspinous implants on flexibility and intradiscal pressure. Eur Spine J 2008;17:1049-56.

48. Anasetti F, Galbusera F, Aziz HN, et al. Spine stability after implantation of an interspinous device: an in vitro and finite element biomechanical study. J Neurosurg Spine 2010;13:568-75.

49. Alfieri A, Gazzeri R, Prell J, et al. Role of lumbar interspinous distraction on the neural elements. Neurosurg Rev 2012;35:477-84.

50. Schulte LM, O’Brien JR, Matteini LE, Yu WD. Change in sagittal balance with placement of an interspinous spacer. Spine (Phila Pa 1976) 2011;36:E1302-5. 